\title{
Positron Annihilation Methods by Use of LSO Scintillators
}

\author{
T. HIRADE ${ }^{a b, *}$ AND T. OKA ${ }^{c, d}$ \\ ${ }^{a}$ Nuclear Science and Engineering Directorate, Japan Atomic Energy Agency, Tokai, Ibaraki, 319-1195, Japan \\ ${ }^{b}$ Institute of Applied Beam Science, Graduate School of Science and Engineering, Ibaraki University \\ 4-12-1 Nakanarusawa, Hitachi, Ibaraki, 316-8511, Japan \\ ${ }^{c}$ Center for the Advancement of Higher Education, Tohoku University \\ 41 Kawauchi, Aoba-ku, Sendai, Miyagi, 980-8576, Japan \\ ${ }^{d}$ Department of Chemistry, Graduate School of Science, Tohoku University, 6-3 Aoba, Aramaki \\ Aoba-ku, Sendai, Miyagi, 980-8578, Japan
}

\begin{abstract}
$\mathrm{Lu}_{2} \mathrm{SiO}_{5}$ : Ce (LSO) scintillator is one of the scintillators used for positron emission tomography because of its high efficiency of gamma-ray detection. We tried to use LSO for positron annihilation lifetime and age-moment um correlation measurements because the high efficiency is needed to reduce the positron irradiation effect during measurements. The high efficiency with reasonable time resolution was successfully obtained by use of the dynode signals from photomultiplier tubes.
\end{abstract}

DOI: 10.12693 /APhysPolA.125.825

PACS: 78.70.Bj

\section{Introduction}

There are several positron annihilation methods that are very unique and strong tools in many different fields. A positron is an antiparticle of an electron, therefore we need to produce positrons. One way to obtain the positrons is by use of radioisotopes and the other is by the pair creation caused by high energy accelerators. Here we concentrate on the methods with the radioisotopes, especially ${ }^{22} \mathrm{Na}$. The most important and often used method is probably the positron annihilation lifetime (PAL) measurement. Even in the case of PAL, the irradiation effects caused by the positron source will affect the experimental results [1]. There are two ways of measuring PAL, dual coincidence and triple coincidence methods. The systems for both of the methods have one detector for $1.27 \mathrm{MeV}$ gamma-ray from ${ }^{22} \mathrm{Na}$ that appears almost simultaneously with the positron injection to samples. The system for the former method has another detector to obtain the time information of the positron annihilation, and the latter system has two other detectors for both of the annihilation gamma-rays. The triple coincidence method can give better time resolution [2].

Positron annihilation age-momentum correlation (AMOC) measurement is also the triple coincidence method, and both of the annihilation gamma-rays are detected. The deference from PAL of the triple coincidence method is one of the detectors for the annihilation gamma-rays, is a high purity Ge semiconductor detector (HPGe). It is possible to say that AMOC is a combination of PAL and measurement of annihilation gamma-ray energy with HPGe, i.e. the Doppler broadening (DB) measurement. Therefore AMOC can give the positron

*corresponding author; e-mail: hirade.tetsuya@jaea.go.jp age dependent annihilation gamma-ray energy, DB, spectra.

AMOC is a very strong tool to investigate many different kinds of materials, such as metals, semiconductors, polymers, and molecular solids and liquids [3-8]. The irradiation effect is probably important for polymers and molecular solids and liquids, i.e. insulating materials. Positrons injected in the insulating materials will form positronium (Ps) at the terminal spur of the positron track where there are many ions, excess electrons and a thermalized positron. The thermalized positron has some possibility to form Ps by capturing one of the excess electrons. The detail is explained by the spur reaction model proposed by Mogensen [9]. Some reactive species formed by the positron irradiation during measurements will affect the Ps formation and reactions.

$P_{s}$ is a bound state of an electron and a positron, then there are two different spin states, one is the singlet Ps (para-Ps) and the other is the triplet Ps (ortho-Ps). Para-Ps and ortho-Ps have 125 ps and 142 ns intrinsic annihilation lifetime in vacuum. $142 \mathrm{~ns}$ is very long and hence the positron in ortho-Ps has very large chance to annihilate with electrons having antiparallel spins of the positron spin in surrounding molecules. This annihilation process is called "pick-off annihilation" and the lifetimes are shortened to $1-5 \mathrm{~ns}$ in condensed materials. Some of the positrons do not form Ps and annihilate with one of the electrons in surrounding molecules with the lifetime of about 400 ps. It is free from Ps formation so-called "free positron" that does not mean a freely moving positron. The momenta of the electron and the positron in para-Ps cancel out and then only the para-Ps intrinsic annihilation gives narrow energy distribution of annihilation gamma-rays.

PAL spectra for insulating materials usually have three lifetime components. However, if there are reactions of ortho-Ps, the lifetime can be shortened additionally to 
pick-off shortening but there is no way to know from the lifetime spectra. On the other hand, the energy spectra at the ortho-Ps annihilation age region must show some information about the reaction of ortho-Ps in the case of AMOC. For example, ortho-Ps and hydroxyl radical reactions make the longest lifetime shorter in the case of water [10]. Hence, the lifetime is shorter at higher temperatures [11]. The evidence of existence of the reactions of ortho-Ps was obtained by AMOC measurement [12].

TABLE I

Characteristics of LSO and $\mathrm{BaF}_{2}$ scintillators [16].

\begin{tabular}{c|c|c|c}
\hline \hline & $\begin{array}{c}\text { Density } \\
{[\mathrm{g} / \mathrm{cm}]}\end{array}$ & $\begin{array}{c}\text { Wavelength } \\
{[\mathrm{nm}]}\end{array}$ & $\begin{array}{c}\text { Decay time } \\
{[\mathrm{ns}]}\end{array}$ \\
\hline $\mathrm{LSO}$ & 7.4 & 420 & 40 \\
$\mathrm{BaF}_{2}$ & 4.89 & 220 & 0.8
\end{tabular}

AMOC is a very strong tool to study reactions of Ps and free positrons as mentioned above, but it is the triple coincidence method and HPGe detectors are very slow and hence the count rate of AMOC is usually very small, i.e. it takes very long time to collect a spectrum. As mentioned above, the irradiation effect by the positron irradiation is not negligible in the case of insulating materials. To obtain the reliable experimental results, we need to have higher count rate for AMOC. We tried to use $\mathrm{Lu}_{2} \mathrm{SiO}_{5}$ : Ce (LSO) scintillators to have high count rate to reduce the irradiation effect caused by the positron source, because LSO scintillator is one of the scintillators used for positron emission tomography (PET) because of its high efficiency of gamma-ray detection [13-15]. The characteristics of LSO and $\mathrm{BaF}_{2}$ scintillators are shown in Table I.

\section{Experimental}

The shape of the LSO scintillators is circular truncated cone with $30 \mathrm{~mm}$ diameter at the top, $40 \mathrm{~mm}$ diameter at the bottom, and $30 \mathrm{~mm}$ height. The scintillators are mounted on photomultiplier tubes, H1949 (Hamamatsu). PAL measurements were performed by use of a set of two detectors. For the comparison, $\mathrm{BaF}_{2}$ scintillators with the exactly same shape mounted on H3378 (Hamamatsu) were also used.

\section{TABLE II}

Characteristics of the photomultiplier tubes.

\begin{tabular}{c|c|c|c}
\hline \hline & $\begin{array}{c}\text { Blue } \\
\text { sensitivity }\end{array}$ & $\begin{array}{c}\text { Rise time } \\
{[\mathrm{ns}]}\end{array}$ & $\begin{array}{c}\text { Dynode structure } \\
\text { /number }\end{array}$ \\
\hline $\mathrm{H}-1949$ & 10.5 & 1.3 & line focus $/ 12$ \\
$\mathrm{H}-3378$ & 10.0 & 0.7 & line focus $/ 8$
\end{tabular}

The characteristics of these photomultiplier tubes are indicated in Table II. For AMOC measurements, the used HPGe was LO-AX (SEIKO EG\&G) type semiconductor detector. The waves from the photomultiplier tubes and the amplified wave by an amplifier (572; Ortec) from the HPGe detector are saved on the hard disk in a digital storage oscilloscope (DSO), Wavepro7100A
(LeCroy) and analyzed afterward. $49 \mathrm{kBq}{ }^{22} \mathrm{Na}$ sandwiched with two $7.5 \mu \mathrm{m}$ Kapton foils was used as the positron source. ${ }^{22} \mathrm{Na}$ emits a $1.27 \mathrm{MeV}$ gamma-ray with emitting a positron. This gamma-ray can be used to know the information of positron injection, i.e. a start signal, by use of one of the scintillation detectors. The other detectors are used for the detection of one of the annihilation gamma-rays, i.e. for a stop signal. In the case of AMOC, HPGe is used to measure the energy of the other annihilation gamma-ray.

We measured two samples in this work. One was Kapton (DuPont) as a standard sample to obtain time resolution, because it has just one lifetime component, $385 \mathrm{ps}$ [17]. The other was a fused quartz that was also a standard sample (NMIJ CRM 5601a) supplied by AIST with a known value of the longest lifetime as $1.62 \mathrm{~ns} \mathrm{[18].}$ The total counts for these measurements were about 1.5 million. The lifetime spectra were analyzed with PALSfit programme [19].

\section{Results and discussion}

Although the PAL apparatus by use of $\mathrm{BaF}_{2}$ scintillators gave $260 \mathrm{ps}$ time resolution (FWHM), the time resolution obtained by just replacing the LSO detectors was about $500 \mathrm{ps}$ (FWHM). Then we tried to improve the time resolution by use of dynode signals from photomultiplier tubes, instead of the anode signals. The dynode signals are obtained from earlier dynode unit and then better time information is expected [20].

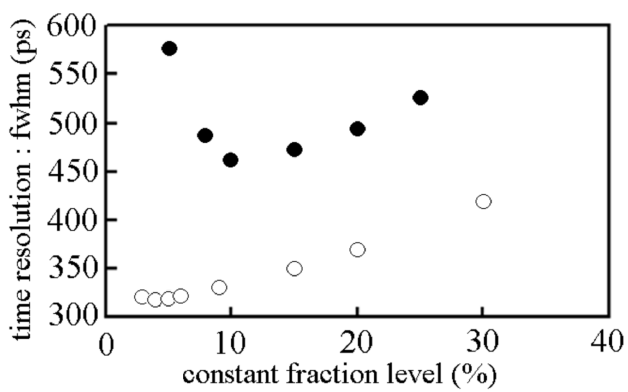

Fig. 1. Constant fraction level dependence on the time resolutions for PAL with two LSO scintillation detectors. Filled circles and open circles indicate results with the signals from the anodes and the dynodes, respectively.

Moreover, the waves from dynodes showed sharp rises therefore the timing information obtained from the crossing point at the $3 \%$ of the height of the peak, i.e. from the constant fraction of $3 \%$, gave better time resolution as indicated in Fig. 1. The sharp rises of the wave signal from the dynode of H1949 could make the time resolution better. Our best time resolution for the PAL apparatus with LSO scintillators was 306 ps with almost twice more count rate of $\mathrm{BaF}_{2}$ apparatus. The three times more count rate was also possible with 323 ps time resolution by applying wider energy window for the stop signal. For PAL, it is difficult to apply wider energy windows for start signal, because of the ${ }^{176} \mathrm{Lu}$ in LSO 
scintillators. $2.59 \%$ of $\mathrm{Lu}$ is ${ }^{176} \mathrm{Lu}$ which is a radioactive element. A LSO scintillator with the shape indicated above has about $9 \mathrm{kBq}$ activity. ${ }^{176} \mathrm{Lu}$ emits beta-ray with the maximum energy of $593 \mathrm{keV}$ and $88 \mathrm{keV}, 202 \mathrm{keV}$ and $307 \mathrm{keV}$ gamma-rays with gamma-ray abundances of $15 \%, 78 \%$ and $94 \%$, respectively. $593 \mathrm{keV}$ beta-ray energy should give a full-absorption signal always and then, for example, if the $202 \mathrm{keV}$ gamma-ray is absorbed in the scintillator, the signal pulse height will be sum of the beta-ray energy and $202 \mathrm{keV}$. If the start detector has a wide energy window for $1.27 \mathrm{MeV}$ gamma-ray and the beta-ray energy is in higher part of the energy distribution, sum of the beta-ray energy and $202 \mathrm{keV}$ can be registered by the start detector. The $307 \mathrm{keV}$ gamma-ray can escape from the scintillator and come into the other scintillator to give a signal suitable for the stop signal, i.e. in a wide energy window for $511 \mathrm{keV}$ annihilation gamma-ray. Hence, the coincidence events caused by ${ }^{176} \mathrm{Lu}$ can come into the lifetime spectra with the wide energy windows for start and stop signal. The ${ }^{176} \mathrm{Lu}$ total energy of beta-ray and high abundance gamma-rays, i.e. $593 \mathrm{keV}, 202 \mathrm{keV}$, and $307 \mathrm{keV}$, is $1102 \mathrm{keV}$ at highest.

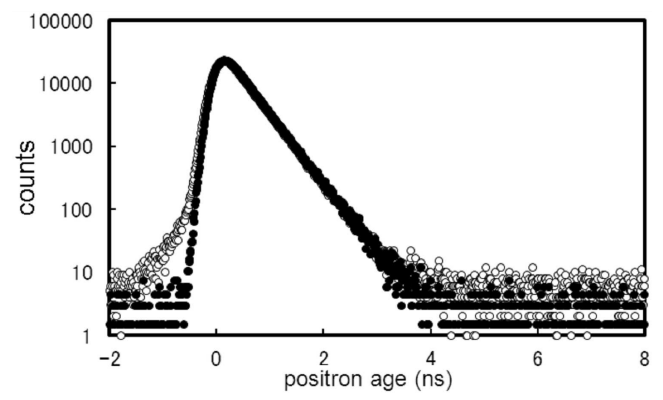

Fig. 2. Lifetime spectra of Kapton. Results depicted as circles were obtained with wide energy windows for start and stop signals. A narrow energy window, i.e. the full absorption peak area, for just start signals was applied to obtain results depicted as filled circles. The values of the filled circles were normalized by the ratio between the total counts for both spectra, 1.476.

Therefore the random coincidence background can be higher for the wide energy window for the start signals indicated in Fig. 2. The narrow energy window for the full absorption peak of the $1.27 \mathrm{MeV}$ gamma-ray from ${ }^{22} \mathrm{Na}$ was acceptable to have suitable lifetime spectra. The results obtained for $\mathrm{BaF}_{2}$ scintillators and LSO scintillators are shown in Table III.

TABLE III

Results obtained for measurements with narrow energy windows.

\begin{tabular}{c|c|c|c}
\hline \hline & $\begin{array}{c}\text { Time resolution } \\
\text { (FWHM) [ps] }\end{array}$ & $\begin{array}{c}\text { Count rate } \\
\text { [cps] }\end{array}$ & $\begin{array}{c}\text { Peak to } \\
\text { background } \\
\text { ratio }\end{array}$ \\
\hline $\mathrm{BaF}_{2} / \mathrm{H}-3378$ & 260 & 18 & 17900 \\
$\mathrm{LSO} / \mathrm{H}-1949$ & 306 & 32 & 16600
\end{tabular}

On the other hand, AMOC measurement is a triple coincidence method and then the effect of ${ }^{176} \mathrm{Lu}$ on the spectra will be very small. Therefore it is possible to apply wider energy windows even for the start signals. Hence the count rate of LSO AMOC apparatus becomes, at least, 5 times greater than that of $\mathrm{BaF}_{2}$ apparatus.

For AMOC measurements, we used the conventional analogue lifetime system with CFDDs (583; Ortec) and a TAC (457; Ortec) to produce the trigger signals for DSO. We used the state qualified trigger method with the trigger signal from the TAC and the amplified wave from HPGe. The qualified triggers are a signals transition above or below a given level as an enabling, i.e. qualifying condition for a second signal that is the trigger source. For the state qualified triggers, the amplitude of the first signal must remain in the desired state until the trigger occurs.

When we use the conventional analogue lifetime system, CFDD just accepts the signals from anodes of the photomultiplier tubes. Connecting the anode to CFDD seemed to affect the dynode signals and then the time resolution became worse. The time resolution with very wide energy windows was about 390 ps.

Lifetime spectrum, energy spectrum and $S(t)$ curve for the standard sample (NMIJ CRM 5602a) are shown in Fig. 3, Fig. 4, and Fig. 5. $S$ values were obtained by the fraction of counts coming into $\pm 3 \times 10^{3} m_{\mathrm{e}} c . m_{\mathrm{e}}$ is the rest mass of a electron and $c$ is the velocity of light. The longest lifetime of the spectrum in Fig. 4 was 1.628 ns that was very close to the value 1.62 ns supplied by AIST. Both of the lifetime spectrum and the energy spectrum were reasonable. Especially, indication of the left part of the lifetime spectra is very important. You can see a sharp drop of the values at the negative positron age region. The extra component appearing here can make the artificial young age broadening [21].

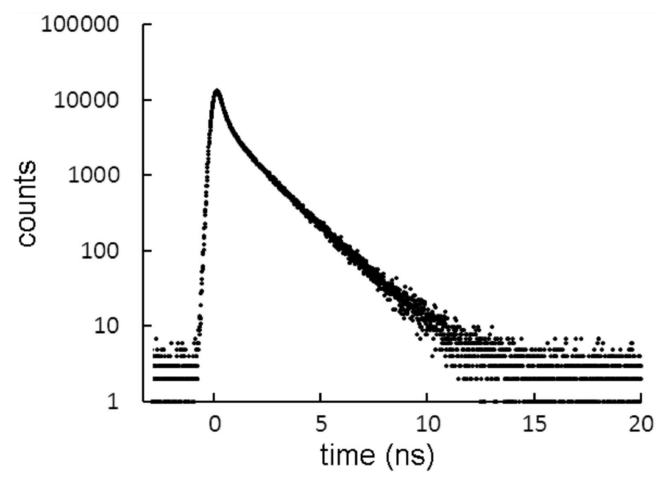

Fig. 3. Lifetime spectrum of the fused quartz (NMIJ CRM 5601a) measured by AMOC with LSO scintillators.

In Fig. 5, $S(t)$ of Kapton was also indicated. Large $S$ values, i.e. narrow energy distributions, appeared at young age region for the materials in which Ps can form. The constant $S$ values for the materials with no Ps formation, such as Kapton, can show the reliability of the 


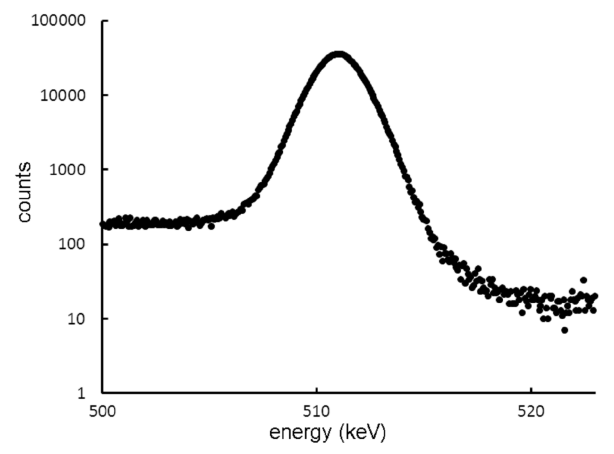

Fig. 4. Energy spectrum of annihilation gamma-rays of the fused quartz (NMIJ CRM 5601a) measured by AMOC with LSO scintillators.

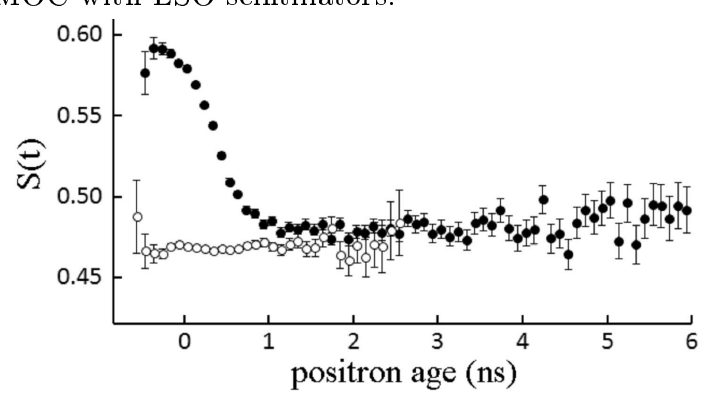

Fig. 5. Positron age dependence of $S(t)$ for the fused quartz (NMIJ CRM 5601a) (filled circles) and the Kapton (open circles) measured by AMOC with LSO scintillators.

apparatus. AMOC is a complicated method and then showing these quite normal results is very important to show that the method applied here is quite reliable. The spectra in Fig. 3 and Fig. 4 and $S$ values in Fig. 5 showed that the AMOC system with LSO scintillators has a very good quality even though we applied wide energy windows for the stop and start detectors. Especially, these results showed that there was almost no effect from ${ }^{176} \mathrm{Lu}$ for AMOC measurements by use of LSO scintillators.

AMOC measurements usually take long time and then the improvement of the count rate by use of LSO scintillators will reduce the irradiation effect caused by the positron irradiation. The count rate after the analysis was about 3 counts/s, i.e. quarter million counts/day, for $49 \mathrm{kBq}^{22} \mathrm{Na}$ source. It is at least about 5 times the one for $\mathrm{BaF}_{2} \mathrm{AMOC}$ systems.

\section{Conclusion}

By use of $\mathrm{Lu}_{2} \mathrm{SiO}_{5}$ : Ce scintillator, we succeeded to obtain high efficiency and reasonable time resolution on the measurements of the positron annihilation methods, such as the positron annihilation lifetime measurement and the age-momentum correlation measurement. It can give smaller change of the sample during measurements caused by the positron irradiation effect. The best time resolution with LSO was $306 \mathrm{ps}$ that is just $50 \mathrm{ps}$ worse than the time resolution, $260 \mathrm{ps}$, with $\mathrm{BaF}_{2}$, by use of the dynode signals. The count rate for AMOC was about
3 counts/s, quarter million counts/day, for $49 \mathrm{kBq}^{22} \mathrm{Na}$ source. It is at least about 5 times the one for $\mathrm{BaF}_{2}$ AMOC systems.

\section{Acknowledgments}

Authors wish to thank Prof. R. Krause-Rehberg and Dr. M. Butterling for a very important suggestion of use of LSO scintillators to improve count rates of AMOC apparatus. This research was supported by a Ministry of Education, Culture, Sports, Science and Technology Grant-in-Aid for Scientific Research (C), 23600011, 20112013.

\section{References}

[1] M. Welander, F.H.J. Maurer, Mater. Sci. Forum 105, 1811 (1992).

[2] H. Saito, Y. Nagashima, T. Kurihara, T. Hyodo, Nucl. Instrum. Methods Phys. Res. A 487, 612 (2002).

[3] K. Inoue, Y. Nagai, Z. Tang, T. Toyama, Y. Hosoda, A. Tsuto, M. Hasegawa, Phys. Rev. B 83, 115459 (2011).

[4] K. Sato, H. Murakami, K. Ito, K. Hirata, Y. Kobayashi, Acta Phys. Pol. A 113, 1511 (2008).

[5] N. Suzuki, T. Hirade, F. Saito, T. Hyodo, Radiat. Phys. Chem. 68, 647 (2003).

[6] T. Hirade, N. Suzuki, F. Saito, T. Hyodo, Phys. Status Solidi C 4, 3714 (2007).

[7] Y. Komuro, T. Hirade, R. Suzuki, T. Ohdaira, M. Muramatsu, Radiat. Phys. Chem. 76, 330 (2007).

[8] T. Hirade, Mater. Sci. Forum 607, 232 (2009).

[9] O.E. Mogensen, J. Chem. Phys. 60, 998 (1974).

[10] S.V. Stepanov, V.M. Byakov, T. Hirade, Radiat. Phys. Chem. 76, 90 (2007).

[11] K. Kotera, T. Saito, T. Yamanaka, Phys. Lett. A 345, 184 (2005).

[12] T. Hirade, T. Oka, J.J. Lee, Mater. Sci. Forum 666 103 (2011).

[13] W.W. Moses, S.E. Derenzo, IEEE Trans. Nucl. Sci. 46, 474 (1999).

[14] M. Moszyński, M. Kapusta, A. Nassalski, T. Szczęśniak, D. Wolski, L. Eriksson, C.L. Melcher, IEEE Trans. Nucl. Sci. 53, 2484 (2006).

[15] M. Moszynski, T. Szczesniak, Acta Phys. Pol. B 4, 59 (2011).

[16] R. Valentini, R. Vianden, Nucl. Instrum. Methods Phys. Res. A 623, 1002 (2010).

[17] T. Hirade, T. Oka, N. Morishita, A. Idesaki, A. Shimada, Mater. Sci. Forum 733, 151 (2013).

[18] K. Ito, T. Oka, Y. Kobayashi, Y. Shirai, K. Wada, M. Matsumoto, M. Fujinami, T. Hirade, Y. Honda, H. Hosomi, Y. Nagai, K. Inoue, H. Saito, K. Sakaki, K. Sato, A. Shimazu, A. Uedono, J. Appl. Phys. 104, 026102 (2008).

[19] P. Kirkegaard, J.V. Olsen, M. Eldrup, N.J. Pedersen, 2009, PALSfit Ris $\varnothing-R-1652(\mathrm{EN})$.

[20] B. Bengtson, M. Moszynski, Nucl. Instrum. Methods 204, 129 (1982).

[21] T. Hirade, Radiat. Phys. Chem. 76, 84 (2007). 